\title{
A HIGH RESOLUTION STUDY OF CONTINUOUS PULSATIONS IN THE EUROPEAN SECTOR
}

\author{
H. W. HANSON and D. C. WEBB \\ Department of Physics, University of York, Heslington, York YO1 5DD, U.K. \\ and \\ D. BEAMISH \\ Institute of Geological Sciences, Murchison House, West Mains Road, Edinburgh EH9 3LA, U.K.
}

DOI 10.1016/0032-0633(79)90060-6

\begin{abstract}
Complex demodulation has been described in detail and applied to Pi2 pulsations in a previous paper by Beamish et al. (1979). The technique is now extended to demonstrate spatiotemporal variations in the fundamental characteristics of $\mathrm{Pc} 3$ and $\mathrm{Pc} 4$ pulsations along a meridional profile extending from the U.K. to Iceland. With the exception of a high latitude Pc4 coupled resonance the results are consistent with a $-90^{\circ}$ Hughes rotation (introduced by the ionosphere) of magnetospheric toroidal line resonances. Furthermore, the ionosphere appears capable of smoothing away the polarisation reversal which would be expected across such amplitude maxima within the plasmasphere. However, a toroidal line resonance in the Pc3 period range about which a sense of polarisation reversal is clearly observed on the ground is suggested as occurring at the plasmapause. This is accounted for in terms of the width of the resonance structure.
\end{abstract}

\section{INTRODUCTION}

The traditional methods of wave analysis (i.e. spectra, hodograms, etc.) by their very nature necessitate some degree of time-averaging and, consequently, obscure any rapid temporal variations in the fundamental parameters defining the waveform in question. In a companion paper (Beamish et al., 1979) the technique of complex demodulation is described and is shown to allow precise determination of the central irequency defining a pulsation event, the temporal characteristics of both amplitude and phase throughout its duration and, therefore, the characteristics of the polarisation parameters of the event. In this previous paper the technique is applied to irregular night-time geomagnetic pulsations and the results compared with current theories of resonating field lines (see Southwood, 1974). Here we apply the same methods to continuous daytime pulsations of longer duration.

Figure 1 shows magnetograms for the two orthogonal horizontal field components $(H$ and $D)$ recorded digitally (with sampling interval $2.5 \mathrm{~s}$ ) on 21 November (day 325) 1977 by the rubidium vapour magnetometers operated under the auspices of the Institute of Geological Sciences on the Faeroes and at Eskdalemuir. These records have been band-pass filtered to exclude periods outside the range $15-100 \mathrm{~s}$ and it is apparent that the remaining signal contains a pulsation with a clearly defined modulation envelope and period slightly longer than $1 \mathrm{~min}$; being observed predominantly in the $H$-component at Eskdalemuir. Figure 2 contains simiiarly treated waveforms recorded immediately after those of Fig. 1. Here the horizontal components show a different dominant period (approx. 40s) which has maximum amplitude in the $H$-component at Faeroes, with the $D$-component again of lesser amplitude. For each of these events simultaneous recordings are available from seven observatories in an approximately meridional profile extending from Iceland to Cambridge. The geographic and geomagnetic coordinates of the stations concerned are contained in Table 1 . This time period was chosen as it provides an opportunity to examine pulsations from both $\mathrm{Pc} 3(10-45 \mathrm{~s})$ and Pc4 (45 150 s) period ranges on either side of local noon.

\section{COMPLEX DEMODULATION APPLIED TO CONTTNUOUS PULSATIONS}

The procedure of complex demodulation allows us to re-examine time-local properties of those variations within selected frequency bands. The demodulated time-series provides instantaneous values of the amplitude and phase of those variations having frequencies within the chosen bandwidth. This process can be resolved into two stages. Figure 3(a) shows the idealised power spectrum of the pulsation to be analysed. The first step is then to establish, within the limits of the spectral resolution, the central period of the event. This is accomplished through a phase analysis of the dominant 
Faeroes
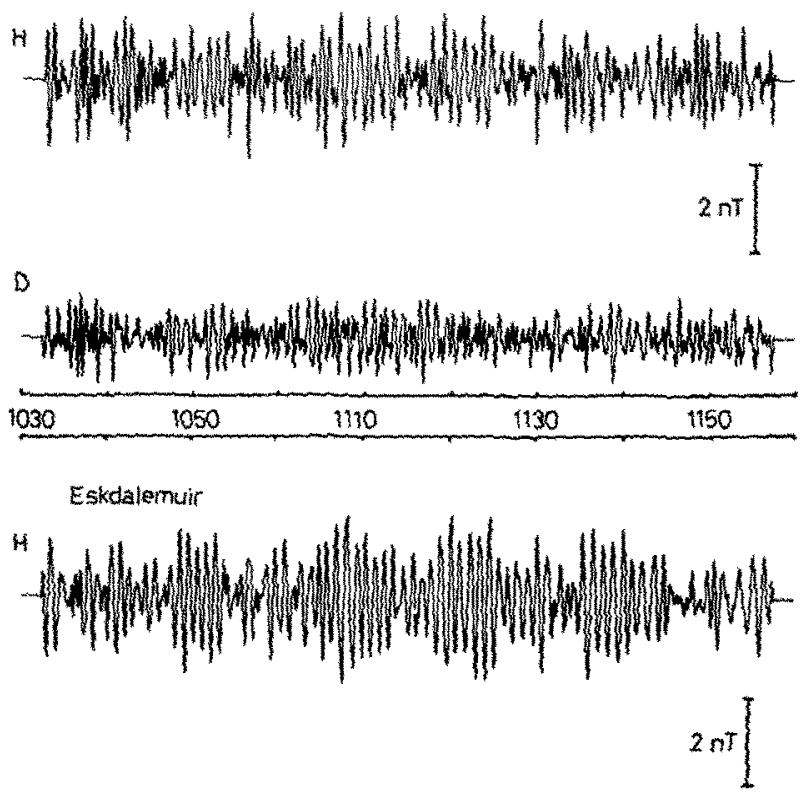

D

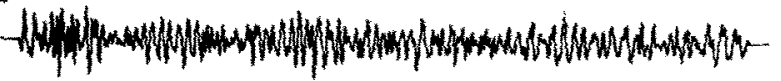
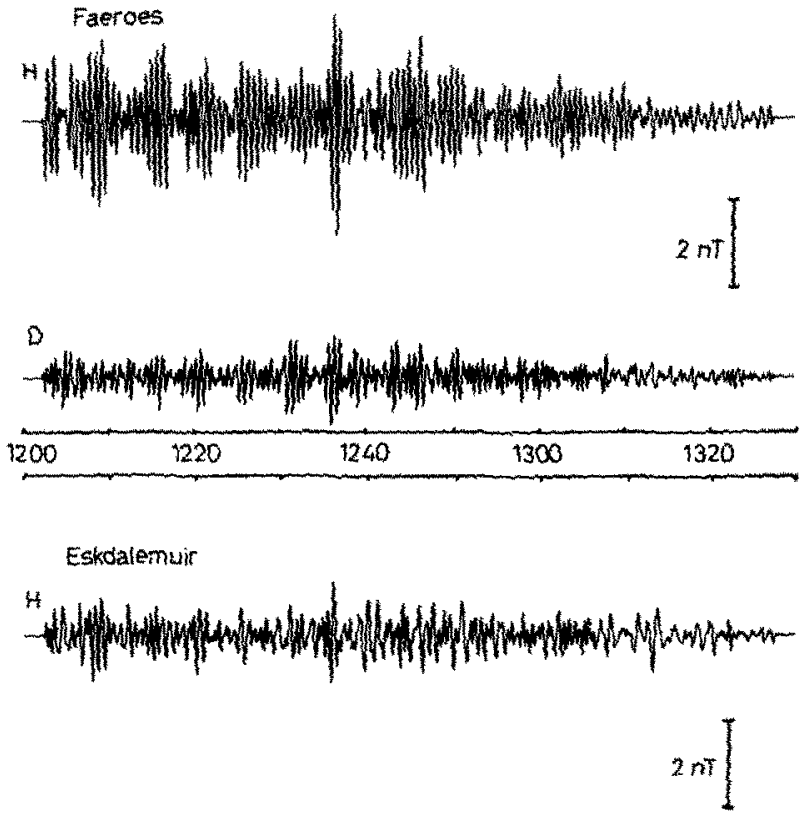

o

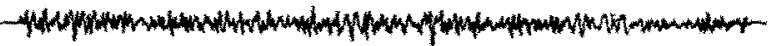

Figs. 1 AND 2. COMPONENTS OF THE GEOMAGNETIC FXELO RECORDED SMULTANEOUSLY AT FAEROES AND ESKDALEMUIR ON 21 NOVEMBER (DAY 325) 1977, gAND-PASS FILTERED BETWEEN 15 AND 100 s. 
TABle 1. Geographic AND geomagnetic STATION COORDINATES

\begin{tabular}{|c|c|c|c|c|c|c|}
\hline \multirow[b]{2}{*}{ Station } & \multirow[b]{2}{*}{ Symbol } & \multicolumn{2}{|c|}{ Geographic coords. } & \multicolumn{2}{|c|}{ Corrected magnetic* } & \multirow{2}{*}{$\begin{array}{c}\text { Corrected } \\
L \text {-value }\end{array}$} \\
\hline & & Lat. & East long. & Lat. & East long. & \\
\hline Eidar & $\mathrm{Eg}$ & 65.4 & 341.0 & 67.23 & 74.47 & 6.68 \\
\hline Reykjavik & Ry & 64.18 & 338.3 & 66.61 & 71.23 & 6.35 \\
\hline Faeroes & $\mathrm{Fa}$ & 62.03 & 353.25 & 61.74 & 81.32 & 4.46 \\
\hline Lerwick & Le & 60.13 & 358.82 & 58.92 & 84.3 & 3.75 \\
\hline I och Laggan & Ll & 56.97 & 355.62 & 56.22 & 80.35 & 3.23 \\
\hline Eskdalemuir & Es & 55.32 & 356.8 & 54.33 & 80.50 & 2.94 \\
\hline York & Yo & 53.95 & 358.95 & 52.62 & 81.58 & 2.71 \\
\hline Cambridge & $\mathrm{Ca}$ & 52.2 & 0.1 & 50.59 & 81.85 & 2.48 \\
\hline Tromso & $\mathrm{Tr}$ & 69.66 & 18.95 & 66.24 & 105.39 & 6.16 \\
\hline Oulu & $\mathrm{Ou}$ & 65.10 & 25.50 & 61.22 & 106.71 & 4.31 \\
\hline
\end{tabular}

* At the Earth's surface for I.G.R.F. (first 7 harmonics) 1975 (computed by M. R. Warner).

component making use of a symmetrical band-pass filter, $W_{p}(f)$, which has a weight of unity only at the central frequency and tapers to zero by the half power points of the spectrum, Fig. 3(b). Now consider equation (7) from Beamish et al. (1979)

$$
\phi_{d}=-\partial \omega . t+\gamma .
$$

Here the temporal variation in the phase of the demodulated series $\left(\phi_{d}\right)$ is expressed in terms of the phase of the original time series $(\gamma)$ and $\partial \omega$, which is the difference between the central frequency of the demodulation band and that of the pulsation event. Thus $\phi_{d}$ will exhibit a linear gradient of $|\partial \omega|$.

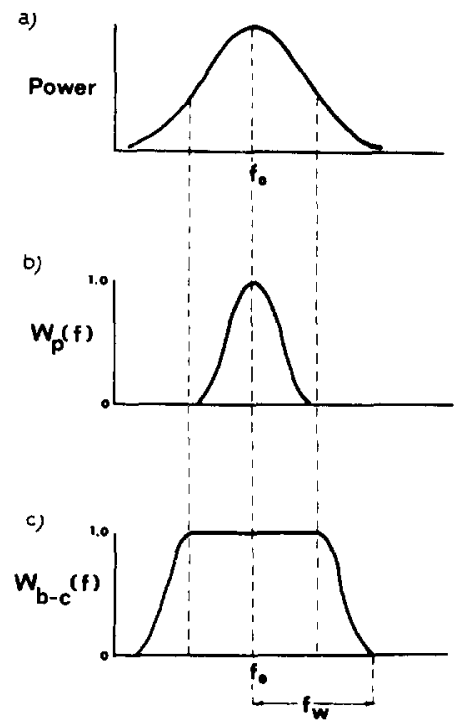

Fig. 3. Filters used IN PHASE ANALYSIS AND DEMODULATION.
The results of this phase analysis for the Pc4 event at Eskdalemuir (Fig. 1) are shown in Fig. 4, from which it is apparent that the gradient of the phase changes sign about a central period of 66.12. Furthermore, the small negative gradient of the $H$-component phase for $66.12 \mathrm{~s}$ suggests that this central period is close to that of the pulsation and it is, therefore, chosen as the centre of the box-car filter, $W_{b c}(f)[F i g .3(c)]$, used in the subsequent demodulation.
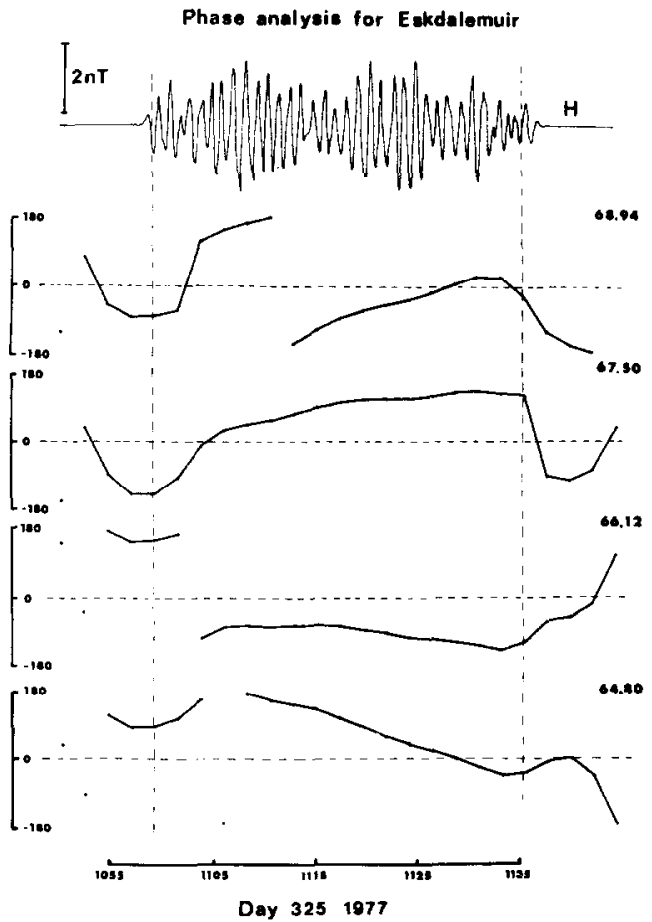

Fig. 4. Phase analysis for Pc4 at eskdalemuir. 
The essential feature of $W_{b c}(f)$ is that the cutoff regions operate outside the half power points of the spectrum and, thus, do not distort the content of the true power spectrum. The other important parameter of the box-car filter is the total half width $f_{w}$, since it is this which determines the effective sampling interval of the demodulated series (Banks, 1975). Consequently, in the demodulation of any piece of data it is essential to quote the central period, the box-car bandwidth and, also, the overall handwidth (including cutoff regions) of the box-car filter.

Following the methodology outlined by Beamish et al. (1979) the same $45 \mathrm{~min}$ section of the Pc4 used in Fig. 4 has been demodulated using a central period of $66.12 \mathrm{~s}$ with box-car bandwidth 54.92 83.08 , total bandwidth $46.96-111.72$, with a resultant effective sampling interval for the demodulated series of $66.12 \mathrm{~s}$. The resulting amplitude and phase of the series are shown below the reproduced horizontal components of the waveform in Fig. 5. It is important to note that as the filter extends to long periods ( $>100 \mathrm{~s}$ ) the data must first be processed to exclude the high power values of these long period components. The 15-100 s band-pass filter already employed is considered adequate. The instantaneous values of the complex demodulates in the $H$

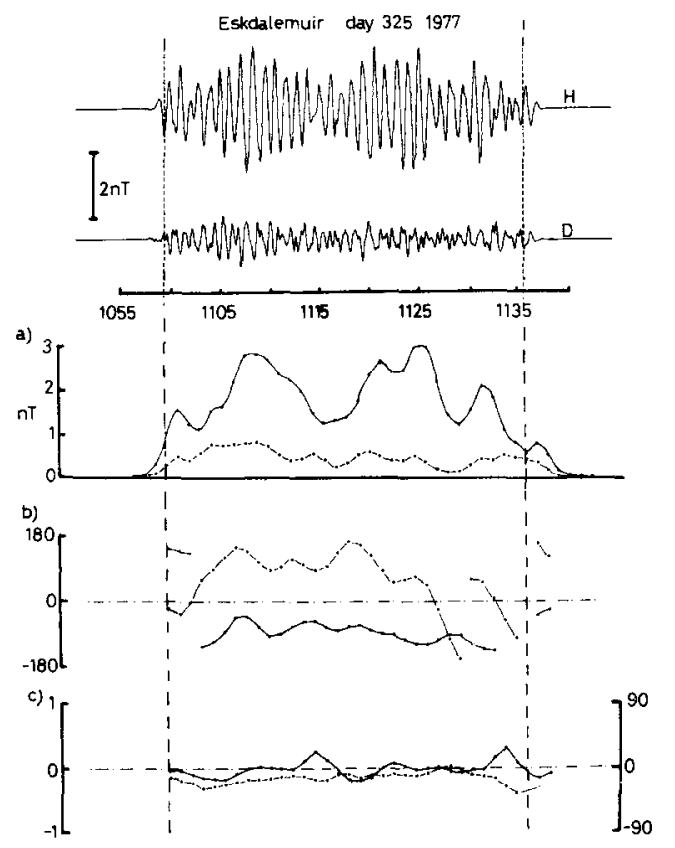

Fig. 5. Results of Demodultation for Pc4 using a CENTRAL PERIOD OF $66.12 \mathrm{~s}$.

(a) Amplitudes; (b) phases; (c) polarisation parameters. and $D$-components can be used to calculate the polarisation characteristics of the horizontal field (Banks, 1975). The resulting temporal variations in the horizontal field ellipticity and ellipse orientation are shown in Fig. 5(c).

The solid line represents the ellipticity in the horizontal plane of the wave polarisation ellipse which is, by definition, positive when there is an anticlockwise sense of rotation looking down in the northern hemisphere. It is apparent that throughout the time interval unaffected by cosine tapering (i.e. within the vertical dotted lines) the ellipticity is very close to zero (linear) whilst the ellipse orientation (dotted line) is also predominantly zero (N-S). This method of analysis has been applied to all stations in the U.K. meridional profile (stations Eidar to Cambridge, Table 1).

An advantage of complex demodulation is that it offers a method of applying a selection criterion in terms of signal to noise ratio. As the rubidium vapour magnetometer is a low-noise device the selection criterion is used to discriminate against magnetospheric rather than equipment noise. In the case of the pre-noon $\mathrm{Pc} 4$, wave estimates with an ellipse semi-major axis amplitude below $0.5 n T$ have been rejected. For the Pc3, however, this amplitude limit results in the rejection of all values for Cambridge. Consequently, the criterion for this event is lowered to $0.25 n T$. In addition, a Hanning procedure using weights $0.25,0.5,0.25$ has been applied to the raw demodulates to improve their stability (Beamish et al., 1979).

In the present study we attempt to combine variations in space and time so that any single parameter of an event in a particular bandwidth (e.g. wave ellipticity) may be presented in latitudetime space, thus providing the maximum information in a single figure. The computational implementation simply involves the definition of a two-dimensional matrix; the columns of which represent instantaneous values of the parameter for all latitudes within the array, whilst the rows of the matrix contain the temporal variations derived via the band-passed demodulated series.

This form of analysis necessarily assumes all stations lie on the same geomagnetic meridian. Since the pulsations are clearly in evidence in the Scandinavian meridian (Fig. 10) and, therefore, do not appear localised in longitude this assumption is considered valid. The spatial resolution is predetermined by the station latitudes given in Table 1 , while the temporal resolution is controlled by the demodulation bandwidth. We first examine the wave characteristics of the pre-noon, longer period 
Pc4 pulsation event and, secondly, the shorter period Pc3 event occurring primarily after midday.

\section{WAVE ChaRACteristics OF Pc3 AND Pc4}

\subsection{Pc4 1055-1140}

Figure 6 is an example of this technique applied to the same time period and using the same band of

(a)

4 AMPLTTUE

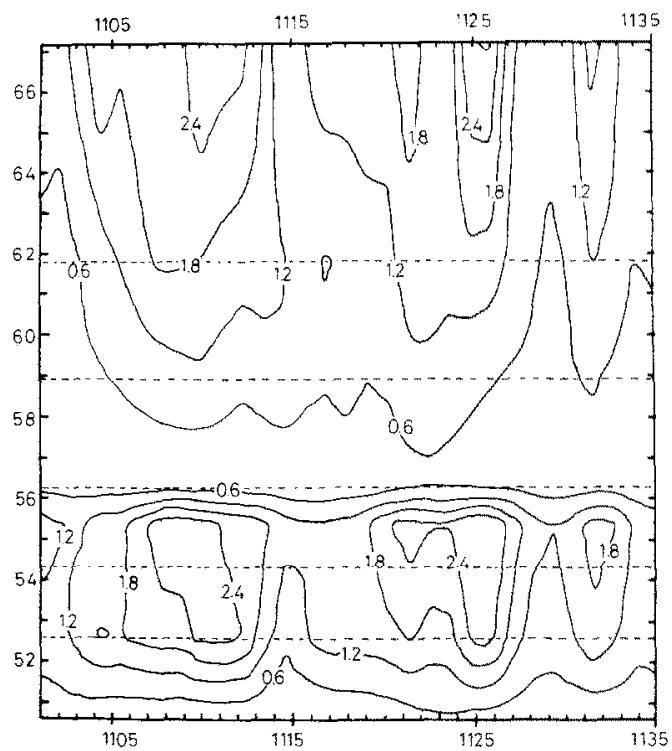

(c)

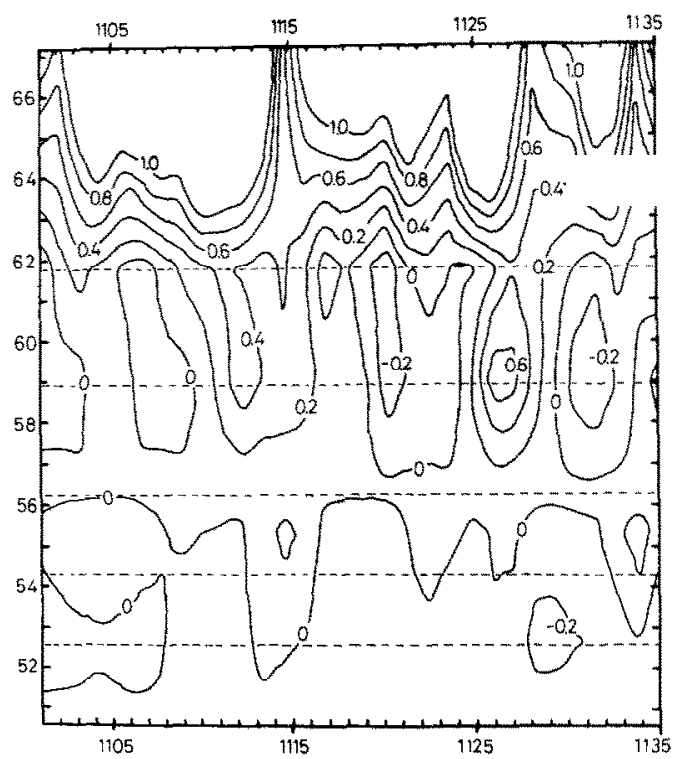

demodulation as shown in Fig. 5; the convention adopted being to represent time by the abscissa and latitude by the ordinate axes. The positions of the stations used are indicated by horizontal dotted lines at the appropriate latitudes. The spatiotemporal variation in the amplitude of the $\boldsymbol{H}$ component is shown in Fig. 6(a). Two peaks (indicating values greater than $2.4 n T$ ) are evident in

(b)

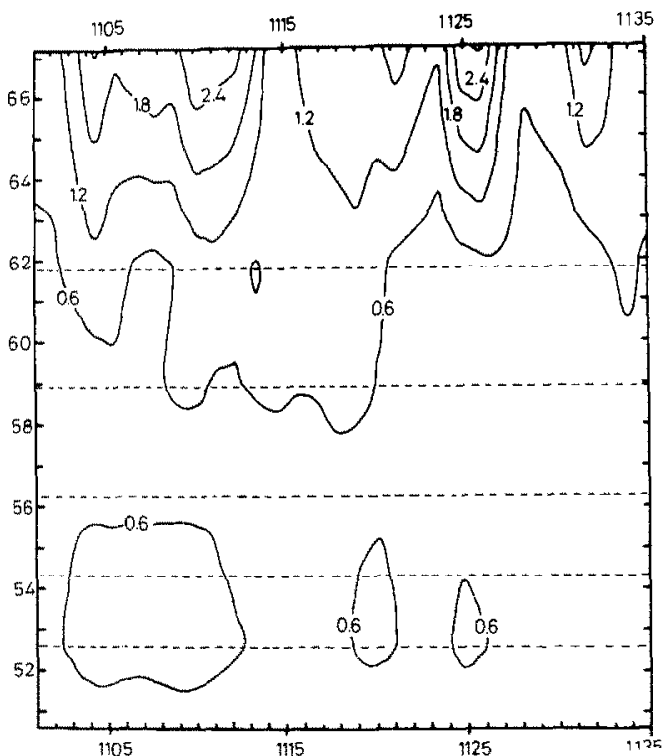

(d)

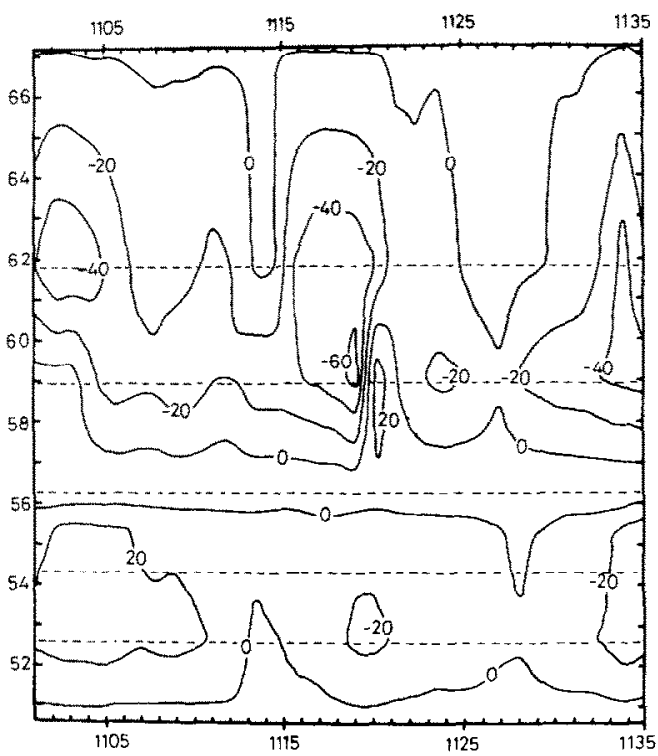

FIG. 6. CONTOUR MAPS USING DEMODULATION BAND CENTRED ON $66.12 \mathrm{~s}$ WITH OVERALL BANDWIDTH 46.96-111.72s.

Effective sampling interval $=66.12 \mathrm{~s}$. 
(a)

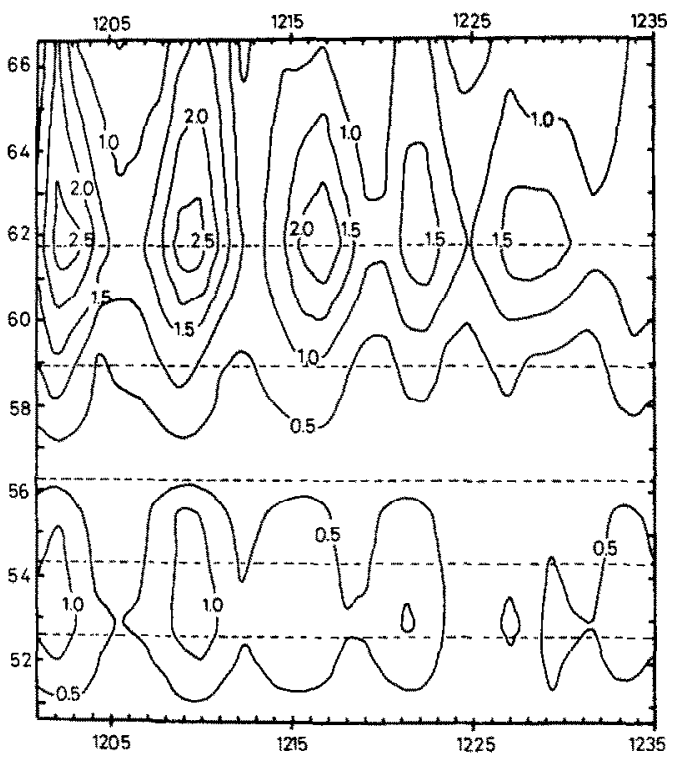

(c)

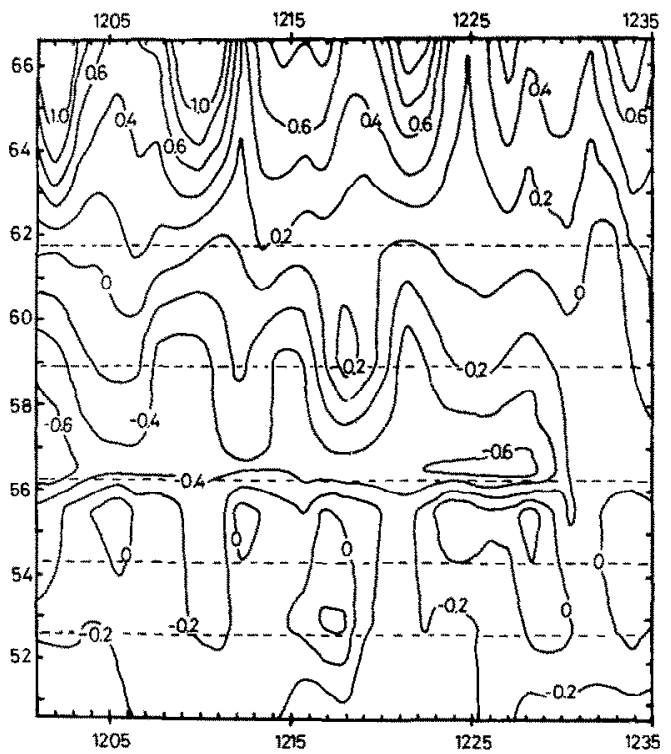

(b)

- ampltTUdE

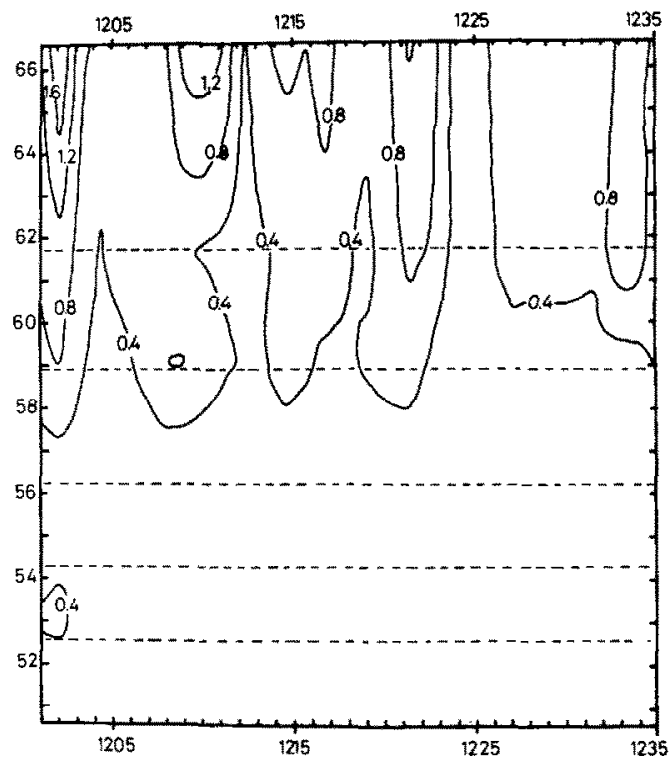

(d) ELLPSE ORIENTATION

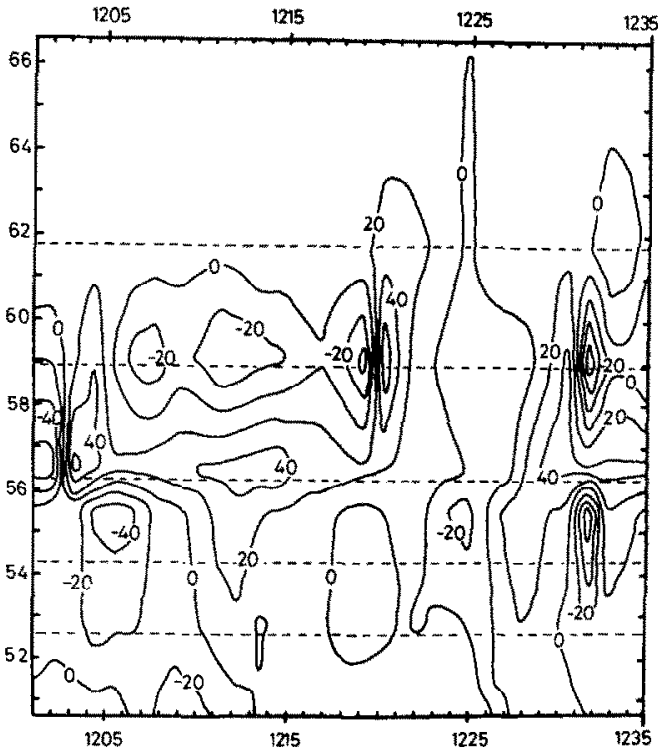

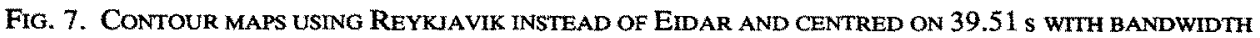
$31.76-52.26 \mathrm{~s}$.

Effective sampling interval $=66.12 \mathrm{~s}$. 
the lower half of the diagram, centred on the geomagnetic latitude of $54^{\circ}$. Table 1 shows that these two peaks observed in the modulation envelope occur at Eskdalemuir. To the south of this latitude the amplitude of the $\boldsymbol{H}$-component falls slowly and steadily whereas to the north the decline is extremely rapid reaching a minimum by approximately $56^{\circ}$ or Loch Laggan and extending as far north as Lerwick (or $59^{\circ}$ ). Continuing further north the amplitude increases once again and there may be a further increase to the north of Eidar, i.e. beyond the limits of the magnetometer array. The D-component [Fig. 6(b)], however, whilst demonstrating an increasing amplitude from the south toward Eidar, does not exhibit such a strong maximum at Eskdalemuir as observed in $H$. However, values exceeding $0.6 n T$ are observed at both Eskdalemuir and York at the appropriate times.

Turning to Fig. 6(c) it is immediately apparent that between York and Eskdalemuir [the location of the amplitude maximum, Fig. 6(a)] the ellipticity is predominantly zero-indicating a linear ellipse. In fact, the values of the ellipticity do not deviate significantly from this value in the entire lower half of the figure. Proceeding northward into the region of rising amplitude, however, the ellipticity increases dramatically becoming anticlockwise and circular.

From Fig. 6(d) the ellipse orientation angle lies between zero and $-20^{\circ}$ (NW) throughout the Eskdalemuir amplitude maximum, only exceeding $-20^{\circ}$ during times of low amplitude. To the north of $57^{\circ}$ latitude the ellipse is orientated in the NW quadrant for all local times reaching values in excess of $-40^{\circ}$ between $59^{\circ}$ and $63^{\circ}$ i.e. from Lerwick to slightly north of Faeroes. Finally, toward the higher latitude amplitude maximum (at or north of Eidar) the ellipse orientation contours suggest a N-S azimuth. However, as the ellipticity in this region of latitude-time space is close to circular [Fig. 6(c)] such an orientation is ill-defined.

\subsection{Pc3 $1155-1240$}

We now consider the $\mathrm{Pc} 3$ pulsation observed immediately after noon on the same day, the central period of which has been determined as $39.51 \mathrm{~s}$ using the phase analysis described above. As before, Fig. 7 , represents complex demodulation "maps" for the first 45 min of the event, using a demodulation band centred on $39.51 \mathrm{~s}$ with box-car from 35.22 to $45.00 \mathrm{~s}$ and overall filter width $31.76-52.26 \mathrm{~s}$. It is important to note that for this part of the analysis the record for Eidar appears noisy and, consequently, Reykjavik has been substituted as the most northerly station-although it lies a little to the west of the meridional profile. Figure 7 (a) clearly demonstrates the existence of two amplitude maxima each fully resolved by the latitudinal array of stations. The northern maximum exhibits five peaks with amplitudes on occasion exceeding $2.5 n T$ at a latitude corresponding to Faeroes $\left(61.7^{\circ}\right)$. To the north and south of this position the amplitude falls smoothly reaching minimum values below $0.5 n T$ between $56^{\circ}$ and $58^{\circ}$ of latitude, just as for the $\boldsymbol{H}$-component amplitude of the Pc4 discussed above. Similarly, a lower latitude maximum is evident within the same latitude range as the $\mathrm{Pc} 4$ although, on this occasion, the amplitude rarely exceeds $1 n T$.

The $D$-component amplitude, however, [shown in Fig. 7(b)] exhibits a very different pattern of spatio-temporal variation in that both the lower latitude maximum and the maximum observed at Faeroes in $H$ are absent. Instead, the amplitude below $59^{\circ}$ is consistently less than $0.4 n T$ whilst to the north of this latitude the $D$-component amplitude increases steadily, reaching values in excess of $1.2 n T$ at Reykjavik, and may continue beyond the northerly limit of the magnetometer array.

In Fig. 7(c) there is clear evidence of a polarisation reversal about Faeroes. To the north of approximately $61^{\circ}$ the ellipticity contours are all positive, indicating an anticlockwise sense of rotation with the more circular polarisations toward the northerly limit of $66.6^{\circ}$. On the other hand, to the south of $61^{\circ}$ the contours are predominantly negative (clockwise). In fact, south of the zero contour (linear ellipticity) the negative values become larger (approaching circular) reaching values of -0.6 in the vicinity of Loch Laggan. Then, proceeding further south, the ellipticity tends rapidly toward zero and remains close to linear throughout the latitude range of the aforementioned secondary maximum.

The pattern of the ellipse orientation variation, Fig. 7(d), can be divided into three distinct latitudinal regions. Firstly, above $61^{\circ}$ latitude the ellipse is confined to orientations close to zero $(\mathrm{N}-\mathrm{S})$. This is significant in the region of the Faeroes maximum, of course, but to the north where the ellipticity approaches circular [Fig. 7 (c)] an orientation of zero is ill-defined. Thus, the best definition for this region is very close to zero at the amplitude maximum and positive to the north. The second region of interest is between the latitudes of the maxima inferred by the $H$-component amplitude, i.e. between $61^{\circ}$ and $54^{\circ}$ of latitude. To the south of 
Faeroes, in Fig. 7(d), the orientation becomes negative during times of largest amplitude (up to 1218 U.T.). It then switches quadrant at approximately $57^{\circ}$ latitude exceeding $40^{\circ}$ in azimuth by Loch Laggan, and returns to an almost $\mathrm{N}-\mathrm{S}$ orientation at a latitude of $55^{\circ}$. The third and remaining latitudinal region is then to the south of $55^{\circ}$, i.e. the location of the lower latitude amplitude maximum,

(a)

H AMPLITUDE

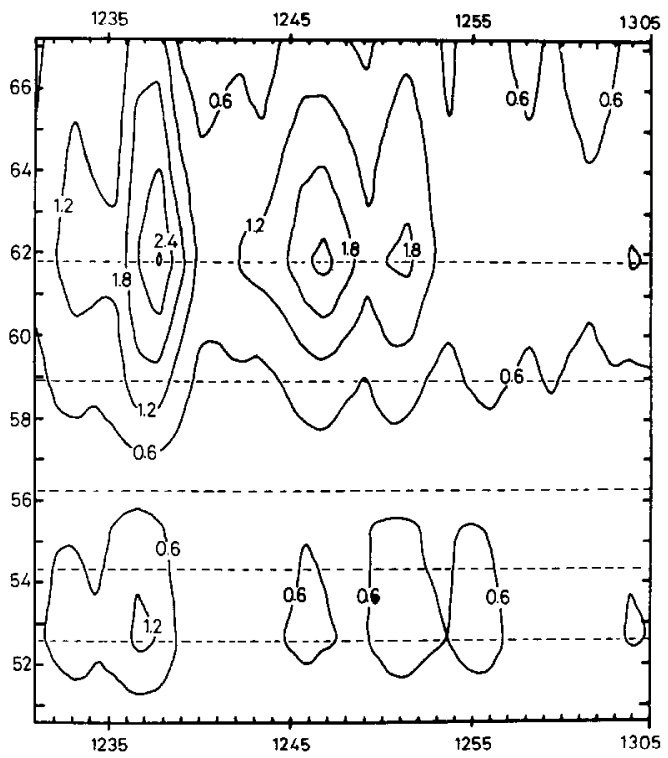

(c)

ELLIPTICITY

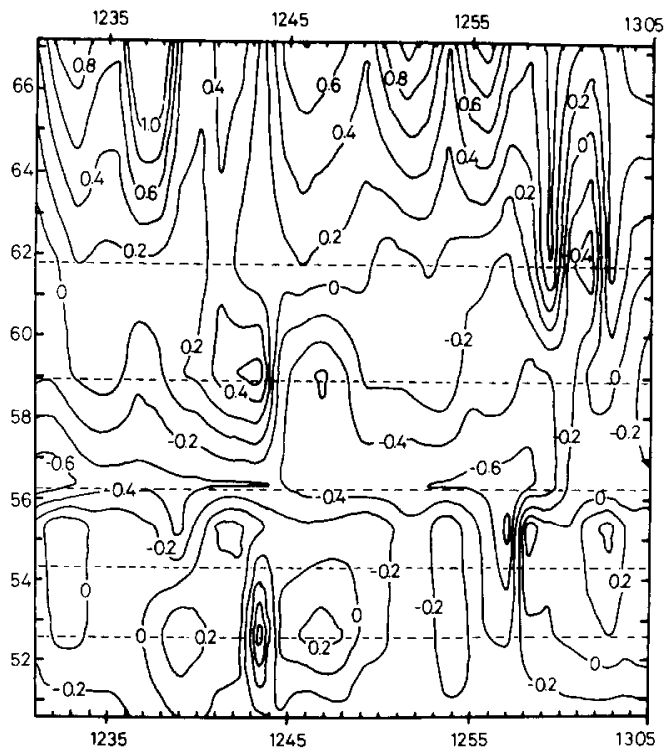

within which the ellipse orientation is predominantly close to zero at times of large $H$-component amplitude [Fig. 7(a)].

\subsection{Pc3 1225-1310}

For completeness, a second $45 \mathrm{~min}$ section of the Pc3 pulsation has been analysed making use of good Eidar data (in place of Reykjavik) once again

(b)

D AMPLITUDE

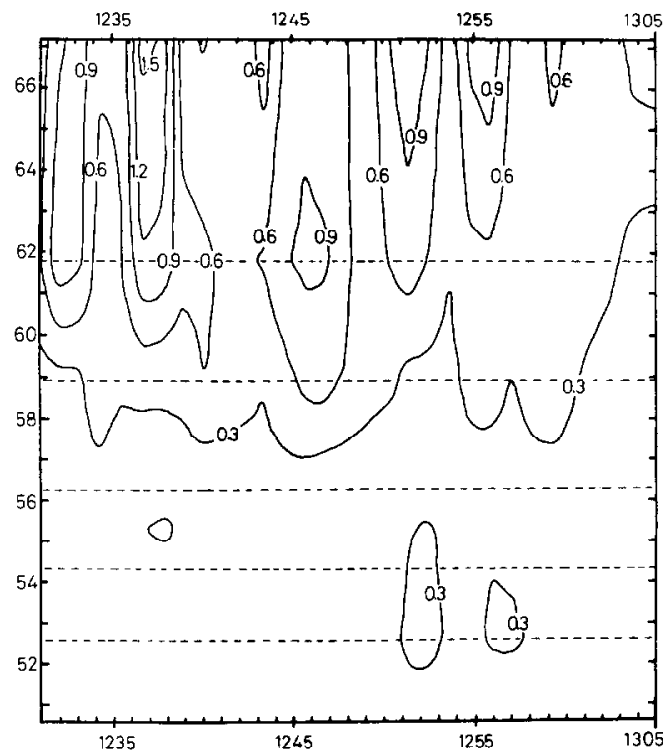

ELLIPSE ORIENTATION

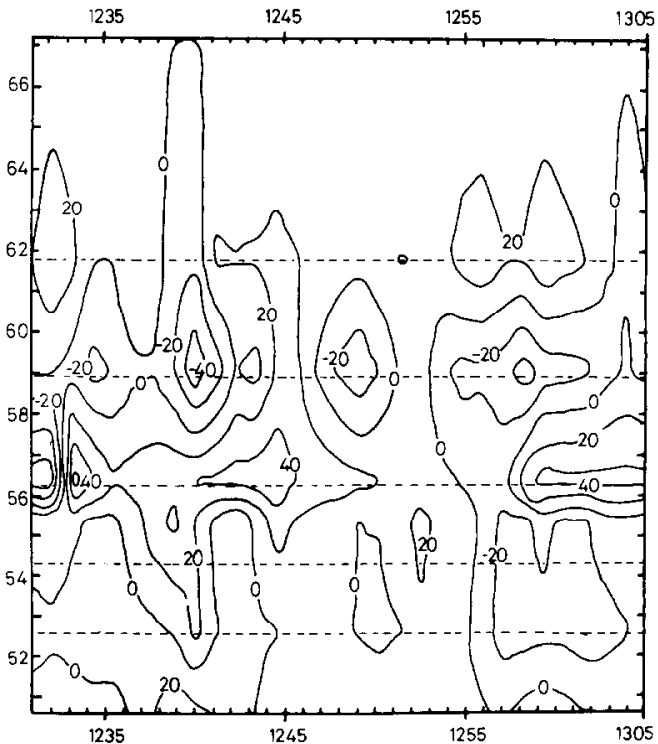

Fig. 8. Contour maps as in Fig. 7 but using Eidar in PLACE of Reykuavik. 
and the demodulation maps are shown in Fig. 8 . The amplitude pattern [Figs. $8(\mathrm{a}, \mathrm{b})$ ] is similar to that of the previous section with resonance at Faeroes and a secondary maximum between York and Eskdalemuir. The $D$-component once again exhibits a pattern of increasing amplitude toward the northerly limit of the array and the absence of well-defined amplitude maxima.

Turning to ellipticity [Fig. 8(c)] a reversal about the amplitude maximum at $61^{\circ}$ is clearly seen with large negative values attained close to Loch Laggan and zero contours in evidence in the vicinity of the lower latitude secondary maximum. Figure 8(d) completes the picture by confirming the pattern observed in Fig. 7(d). That is, an orientation close to zero in the regions of both amplitude maxima with an area between in which the orientation to the south of Faeroes becomes first negative, then zero, then positive before reaching the latitude of the secondary maximum.

\section{DISCUSSION}

The latitude range covered by this study includes stations within the plasmasphere and within the plasmatrough. The boundary between these two regions (i.e. the plasmapause) has been examined in some detail by Chappell et al. (1971) using 150 OGO-5 crossings. Their results indicate that the position of this density discontinuity on the dayside is determined by the level of magnetic activity $\left(K_{p}\right.$ index) during the corotation of that particular sector of the plasmasphere through the nightside region. Once the sector has corotated past dawn the plasmapause position does not respond immediately to changes in the level of activity. Thus, using the average night-time $K_{p}$ value (21000600 L.T.) in equation (2) from Orr and Webb (1975), i.e.

$$
L_{p p}=6.52-1.44 \bar{K}_{p}+0.18 \bar{K}_{p}^{2},
$$

yields a plasmapause position for day 3251977 of $4.76( \pm 0.4) R_{e}$ or between Faeroes and Iceland.

Figure 9 , however, reproduced from the same paper demonstrates the variation in the average plasmapause position with local time for an average $K_{p}$ of 2 and 1.5 as used in the equation above. From this figure it is apparent that the plasmapause moves to larger $L$-values between 0800 and 1800 L.T. and for an average $K_{p}$ of 1.5 the noon position of the plasmapause should be approximately $5.25(+0.4) R_{e}$.

A considerable amount of published work has investigated the relationship between magneto-

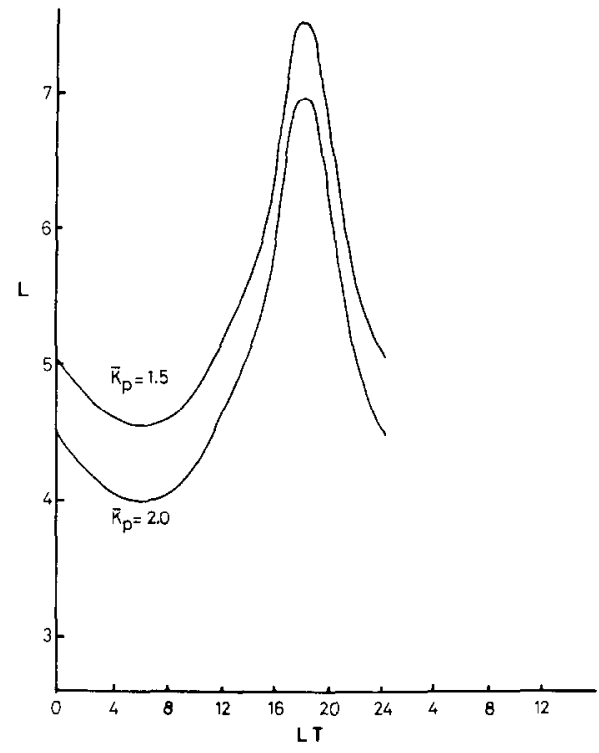

Fig. 9. Variations in aVerage plasmapause position (ORR AND WEBB, 1975).

spheric plasma density gradients and the generation and morphology of pulsations. The recent theories of pulsation structure advanced by Chen and Hasegawa (1974a, b) and Southwood (1974) require an approximately monochromatic wave to couple to a local field line thereby generating a magnetospheric transverse resonance. Studies of Pc3 and Pc4 pulsations near $L=4$ (Lanzerotti et al., 1973; Fukunishi and Lanzerotti, 1974) have shown that dayside pulsation amplitudes are strongly localised in latitude, the latitude of maximum amplitude being a function of wave period. The results contained in these papers also reveal a sense-of-polarisation reversal (change in sign of ellipticity) across the latitude of maximum amplitude, together with a sense-of-polarisation reversal about local noon. The results of Lanzerotti et al. (1973) and Fukunishi and Lanzerotti (1974) all appear consistent with the predictions of the cited theory but indicate that it is the plasma density gradient at the plasmapause that defines the resonance region, i.e. the location of maximum amplitude and linear polarisation of the horizontal field vector. To either side of the resonance region, the amplitude decays smoothly and the coupled wave components (i.e. $H$ and $D$ ) form a vector with circular rotation.

Orr (1975) considers that for impulsively excited events, the usually sharp density gradient at the plasmapause is likely to produce the observed transverse resonance. However, he considers that 
for lightly damped continuous pulsations the plasmapause density gradient may be too abrupt to allow the transverse resonance to develop at the plasmapause. Summarising a variety of statistical $\mathrm{Pc} 3$ and Pc4 results he concludes that, for continuous undamped dayside pulsations, line resonances within the plasmatrough and plasmasphere are the more likely to occur. The uncoupled toroidal line resonance will be characterised by a meridional standing wave structure in the $H$-component (allowing for ionospheric modification, see below).

More recently Green (1978) has studied lightly damped Pc4 pulsations observed within the plasmasphere. His results are consistent with the pulsations being the result of toroidal line resonances within the plasmasphere. In addition, the results of Green (1978) and also Beamish et al. (1979) point to the necessity of taking into account the ionospheric modification of a magnetospheric source signal as formulated by Hughes and Southwood $(1976 \mathrm{a}, \mathrm{b})$. One of the possible consequences of ionospheric modification is a loss of the sense-ofpolarisation reversal across the resonance. Green (1978) points out that although the ionosphere may be incapable of smoothing away a polarisation reversal at the plasmapause, where the density gradient may be discontinuous, it might be capable of doing so within the plasmasphere.

We next consider the results obtained in the light of this discussion assuming throughout the time interval a plasmapause position between Faeroes and Iceland.

\subsection{Pc4 1055-1140}

In Fig. 6(a) we observe a consistent minimum in the $\mathrm{H}$-amplitude between the latitudes of Loch Laggan and Lerwick. To the north and south of this feature resonance maxima are clearly observed. The northern maximum, although not fully defined by the present array, can be safely considered a plasmatrough resonance, whilst the southern maximum in $H$ (at approximately Eskdalemuir) must be plasmaspheric. Comparison of the $H$ and $D$ components in Figs. 6(a) and (b) suggests that the $\mathrm{Pc} 4$ plasmatrough amplitude maximum is a coupled transverse resonance, with comparable amplitudes in both horizontal components. This is supported by the occurrence of circular ellipticity [Fig. 6(c)]. Such an effect would be expected since the toroidal line resonance requires axial symmetry and the departure from dipolar symmetry on the dayside increases with latitude. On the other hand, ellipticity of zero (linear polarisation) within the plasma- sphere in Fig. 6(c) and ellipse orientations of less than $20^{\circ}$ during the amplitude peaks in Fig. 6(d) are indicative of a plasmaspheric toroidal line resonance.

In the case of the fully defined plasmasphere resonance no clear change in sign of ellipticity [Fig. 6 (c)] is observed to the north and south. Such a result is in accord with the results of Green (1978) and points to ionospheric smoothing.

\subsection{Pc3 1155-1240}

In Fig. 7(a) two amplitude maxima are observed the more northerly of which has moved to lower latitude with respect to the longer period Pc4 maximum-which is in agreement with the theory of toroidal and poloidal field line resonances (Orr and Matthew, 1971). Comparison of the $H$ and $D$-components in Figs. $7(\mathrm{a})$ and (b) indicates that neither resonance exhibits a significant degree of coupling, both maxima showing a greater tendency toward toroidal field line resonance. The ellipticity [Fig. 7(c)] exhibits near linear polarisation at the latitudes of the $H$-maxima, while the ellipse orientations [Fig. 7(d)] tend to a $\mathrm{N}-\mathrm{S}$ orientation in these regions. It should be noted that in the latter of these figures the orientation exhibits some degree of temporal modulation; such behaviour being clearly derived from the fast modulation envelopes of the Pc3 wave packets [Fig. 7(a)].

In the case of the plasmatrough resonance. a sense of polarisation reversal about the latitude of the $\mathbf{H}$ maximum is observed [Fig. 7(c)]. A straightforward phase analysis between the $H$ components recorded at Faeroes $(\mathrm{Fa})$ and Oulu (Ou) (the resonant latitude) is shown in Fig. 10. From this it is immediately apparent that Faeroes is leading Oulu in phase, indicating an eastward propagating disturbance, which is in agreement with the observation of an anticlockwise sense of rotation to the north of $61^{\circ}$ and vice versa to the south (Southwood, 1974). In the light of the previous discussion we must consider that ionospheric screening is insufficient to smooth away this reversal in ellipticity observed for the $\mathrm{Pc} 3$ plasmatrough resonance.

Two aspects of $\mathrm{Pc3}$ pulsations can be invoked to explain the observed reversal about the higher latitude resonance. The first concerns the width of the magnetospheric resonance structure which is primarily a function of plasma density gradient and could be sufficiently small such that ionospheric screening is insufficient to remove the spatially rapid Pc3 variation in ellipticity. Furthermore, since the average previous night-time $K_{p}$ is less than 2 


\section{H Components}

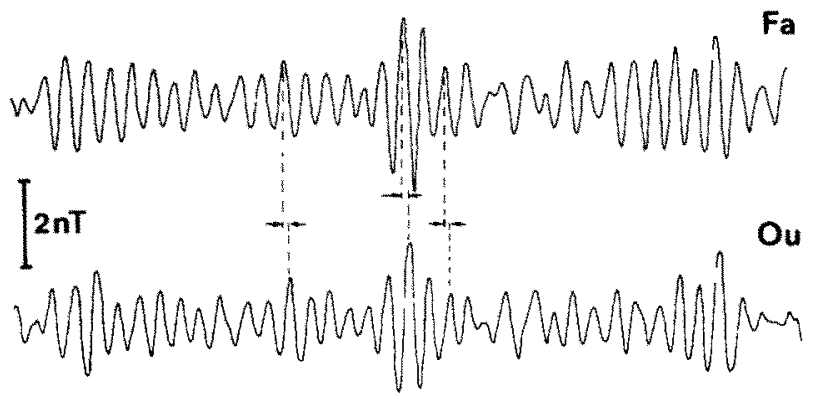

$\begin{array}{llllll}1225 & 1230 & 1235 & 1240 & 1245 & 1250\end{array}$

Fig. 10. Phase analysis between Faeroes and Oulu.

the plasmapause will take the form of a diffuse shallow density gradient possibly capable of supporting a toroidal line resonance. The second characteristic concerns the faster (cf. Pc4) temporal damping of $\mathrm{Pc} 3$ pulsations. It could well be that what we have interpreted as a plasmatrough resonance, only slightly removed from the proposed plasmapause position, is in fact a plasmapause resonance initiated by the higher degree of damping of Pc3 pulsations and the shallow density gradient. This second characteristic would seem the more plausible since we would expect that the resonance structure deriving from the large density gradient at the plasmapause would result in a spatially small resonant structure capable of transmitting a reversal in the sense-of-polarisation through the ionosphere.

\subsection{Pc3 1225-1310}

The spatio-temporal variations in the fundamental parameters of the second section of the $\mathrm{Pc} 3$ confirm the suggested mechanism of a double toroidal line resonance. From Figs. 8(a) and (b) it is apparent that the $H$ and $D$-components are uncoupled and that the positions of the amplitude maxima remain unchanged. The ellipticity reversal about the higher latitude resonance contained in Fig. 7(c) is repeated in Fig. 8(c) which again shows no lower latitude reversal. Similarly, the values of the ellipse orientation [Fig. 8(d)] are consistent with a model of a toroidal line resonance at the plasmapause and a secondary lower latitude plasmaspheric line resonance. These figures serve to reinforce the discussion of Section 4.2 by demonstrating that no discrepancy arises upon substitution of Reykjavik for Eidar.

\section{CONCLUSTON}

The technique of complex demodulation has been shown to offer increased temporal resolution and to facilitate examination of rapid variations in parameters such as ellipticity and ellipse orientation. Making use of the extensive latitudinal array of I.G.S. magnetometers, contour maps (based on band-passed demodulated time series) represent a logical extension of this new method of wave analysis and take full advantage of the increased spatial as well as temporal resolution.

Using an empirical result to estimate the position of the plasmapause, the results obtained have been compared to the development of field line resonances within the plasmasphere, at the plasmapause and within the plasmatrough. The results have also been interpreted in terms of recent predictions of the effects of the ionosphere on groundbased observations.

In the case of $\mathrm{Pc} 4$ pulsations, the results indicate the development of both plasmasphere and plasmatrough resonances. Two resonance regions are also associated with the shorter period Pc3 pulsations. These occur at lower latitudes than the longer period resonances in accord with the theory on toroidal field line resonances. One such resonance occurs close to the inferred position of the plasmapause.

The plasmaspheric resonances of both $\mathrm{Pc} 3$ and $\mathrm{Pc} 4$ waveforms are predominantly uncoupled and occur in the $\boldsymbol{H}$-components. Such behaviour is 
consistent with a $-90^{\circ}$ rotation of a magnetospheric toriodal field line resonance introduced by the ionosphere. The absence of a reversal in the sense of polarisation about the amplitude maximum in the case of the plasmaspheric resonances is similarly consistent with ionospheric screening (Green, 1978).

The higher latitude plasmatrough resonance of the $\mathrm{Pc} 4$ pulsation is observed to be highly coupled whereas that of the $\mathrm{Pc} 3$ exhibits characteristics of a toroidal line resonance. In the case of the shorter period $\mathrm{Pc} 3$ resonance, a reversal in the sense of polarisation about the amplitude maximum is observed. In considering the illustrative models of Hughes and Southwood (1976 b) the ionospheric transmission of this polarisation reversal can be accounted for by a decrease in the width of the resonance structure when compared to that for the longer period $\mathrm{Pc} 4$ pulsation. Such a feature is anticipated since the lateral scale of a resonant structure due to a particular density gradient decreases with both the degree of damping and the period of the waveform.

Finally, it should be mentioned that the plasmapause position based on the empirical model of Orr and Webb (1975) is only used in the absence of a satellite-based density measurement without which any conclusions are only tentative.

Acknowledgements-The authors would like to thank Dr. D. Orr for many helpful comments and the Science Research Council for grant support under contract SGD00178.

This magnetometer project is an IGS/York/Imperial College collaboration in the International Magnetospheric Study and this work was only made possible by the help and diligence of observatory personnel. Our appreciation is extended to U.K. observatory staff (Lerwick, Eskdalemuir), staff at the observatory Nurmijarvi, staff at the Universities of Oulu, Tromso and lceland and staff of the British Antarctic Survey in Cambridge. In particular our thanks go also to Mr. Bjarnason (Eidar), Dr. Saemundsson (Reykjavik), Mr. Apol (Faeroes) and Gordon Duncan (Loch Laggan).

\section{REFERENCES}

Beamish, D., Hanson, H. W. and Webb, D. C. (1979). Complex demodulation applied to $\mathrm{Pi2}$ geomagnetic pulsations. Geophys. J. R. astr. Soc. 58, 471.

Chen, L. and Hasegawa, A. (1974a). A unified theory of long period micropulsations-1. Steady state approach. J. geophys. Res. 79, 1024.

Chen, L. and Hasegawa, A. (19746). A unified theory of long period micropulsations-II. Initial value approach. J. geophys. Res. 79, 1033.

Chappell, C. R., Harris, K. K. and Sharp, G. W. (1971). The dayside of the plasmasphere. J. geophys. Res. 76, 7632.

Fukunishi, H. and Lanzerotti, L. J. (1974). ULF pulsation evidence of the plasmapause--1. Spectral studies of $\mathrm{Pc} 3$ and $\mathrm{Pc} 4$ pulsations near $L=4 . J$. geophys. Res. 79, 142.

Green, C. A. (1978). Meridonal characteristics of a Pc4 micropulsation event in the plasmasphere. Planet. Space Sci. $26,955$.

Herron, T. J. (1966). Phase characteristics of geomagnetic pulsations. J. geophys. Res. 71, 871 .

Hughes, W.J. and Southwood, D. J. (1976a). The screening of micropulsation signals by the atmosphere and ionosphere. J. geophys. Res. 81, 3234.

Hughes, W. J. and Southwood, D. J. (1976b). An illustration of modification of geomagnetic pulsation structure by the ionosphere. J. geophys. Res. 81, 3241 .

Lanzerotti, L. J., Fukunishi, H., Hasegawa, A and Chen, L. (1973). Excitation of the plasmapause at ultralow frequencies. Phys. Rev. Lett., 31, 624.

Orr, D. (1975). Probing the plasmapause by geomagnetic pulsations. Ann. Geophys. 31, 77 .

Orr, D. and Matthew, J. A. D. (1971). The variation of geomagnetic micropulsation periods with latitude and the plasmapause. Planet. Space Sci. 19, 897.

Orr, D. and Webb, D. C. (1975). Statistical studies of geomagnetic pulsations with periods between 10 and $70 \mathrm{~s}$ and their relationship to the plasmapause region. Planet. Space Sci. 23, 1169.

Southwood, D. J. (1974). Some features of field line resonances in the magnetosphere. Planet. Space Sci. 22 , 483. 